\title{
Computational Study of the Energetics and Defect Clustering Tendencies for $\mathrm{Y}$ and La-doped $\mathrm{UO}_{2}$
}

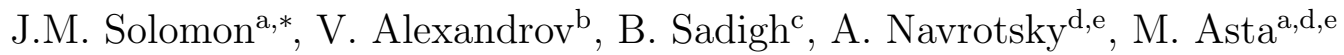 \\ ${ }^{a}$ Department of Materials Science and Engineering, University of California, Berkeley, \\ CA 94720, USA \\ ${ }^{b}$ Physical Sciences Division, Pacific Northwest National Laboratory, Richland, \\ Washington 99352, USA \\ ${ }^{c}$ Physical and Life Science Directorate, Lawrence Livermore National Laboratory, \\ Livermore, California, 94550, USA \\ ${ }^{d}$ Peter A. Rock Thermochemistry Laboratory and NEAT ORU, University of California, \\ Davis, California 95616, USA \\ ${ }^{e}$ Department of Chemical Engineering and Materials Science, University of California, \\ Davis, California 95616, USA
}

\begin{abstract}
The energetics and defect ordering tendencies in solid solutions of fluoritestructured $\mathrm{UO}_{2}$ with trivalent rare earth cations $\left(\mathrm{M}^{3+}=\mathrm{Y}\right.$, La) are investigated computationally using a combination of ionic pair potential and density functional theory (DFT) based methods. Calculated enthalpies of formation with respect to constituent oxides show higher energetic stability for La solid solutions than for Y. Additionally, calculations performed for different atomic configurations show a preference for reduced (increased) oxygen vacancy coordination around La $(\mathrm{Y})$ dopants. The current results are shown to be qualitatively consistent with related calculations and calorimetric measurements of heats of formation in other trivalent doped fluorite oxides, which show a tendency for increasing stability and increasing preference for higher oxygen coordination with increasing size of the trivalent impurity. The implications of these results are discussed in the context of the effect of trivalent impurities on oxygen ion mobilities in $\mathrm{UO}_{2}$, which are relevant to the understanding of experimental observations concerning the effect of trivalent fission products on oxidative corrosion rates of spent nuclear fuel.
\end{abstract}

Keywords: oxygen vacancy, formation enthalpy, pyrochlore, DFT

${ }^{*}$ Corresponding author. email: jsolom@berkeley.edu

Preprint submitted to Acta Materialia

June 9, 2014

(C) 2014. This manuscript version is made available under the Elsevier user license http://www.elsevier.com/open-access/userlicense/1.0/ 


\section{Introduction}

Fluorite-structured oxide compounds can often be doped with high concentrations of trivalent cations. Under reducing conditions this doping leads to the formation of charge compensating oxygen vacancy defects. Recent calorimetry studies on trivalent-cation doped $\mathrm{ZrO}_{2}$ [1], $\mathrm{HfO}_{2}$ [2], $\mathrm{CeO}_{2}$ [3] and $\mathrm{ThO}_{2}$ [4] provide evidence for strong defect association in such systems. The experimental findings have been shown to be consistent with recent computational work on some of these same systems, which demonstrate energetic binding/clustering tendencies between cations and oxygen vacancies, to a degree that has been found to correlate strongly with ionic radii $[5,6,7,8,9,10,11,12]$. Strong oxygen vacancy-cation association reduces the mobility of oxygen ions and therefore the ionic conductivity in aliovalently doped fluorite oxides, which can be detrimental for their use in fuel cell electrolyte and oxygen sensor applications [13].

Similar effects have also been discussed in the context of the electrochemical behavior of spent nuclear fuel composed of fluorite-structured $\mathrm{UO}_{2}$ with dissolved trivalent fission products. Specifically, recent studies have attributed rare earth (i.e., trivalent) fission product dopant-oxygen vacancy clusters to the decreased rate of oxidative dissolution with increasing burnup in spent fuel [14]. The defect clusters are believed to reduce the concentration of mobile oxygen vacancies which facilitate oxidation and eventual dissolution of the fuel, in a manner similar to that by which rare earth doping impedes air oxidation of the cubic fluorite structure to orthorhombic $\mathrm{U}_{3} \mathrm{O}_{8}$ $[15,16,17,18]$. Oxidation and dissolution of nuclear fuel in aqueous environments is of particular concern at the back end of the fuel cycle, i.e., waste disposal [19, 20].

Because of the significant changes in oxidation and corrosion behavior of nuclear fuel associated with rare earth fission products, understanding the thermochemical properties of these defects in $\mathrm{UO}_{2}$ is of particular interest. The effect of rare earth fission product doping on the oxygen chemical potential in $\mathrm{UO}_{2}$ has been studied extensively [21]. However, the energetics and defect ordering tendencies of these systems remain far less studied compared to that for other fluorite-structured oxides $[5,6,7,8,9,10,11,12]$. Specifically, only one calorimetry study [22] and one ionic pair potential modeling investigation [23] have been reported to date. The aim of the current 
work is to provide further insight into the thermochemical behavior of $\mathrm{UO}_{2}$ doped with trivalent cations through the application of density functional theory (DFT) based calculations in which we explore stability and defect ordering/clustering trends with respect to relative cation sizes. It is expected that $\mathrm{UO}_{2}$ substituted with trivalent cations will contain oxygen vacancies or localized electron holes (i.e., $\mathrm{U}^{5+}$ ) as charge compensating defects. The current work focuses only on systems with oxygen vacancies (oxygen deficiency rather than oxygen excess). We consider the smallest and largest trivalent rare earth-fission product cations that are soluble in $\mathrm{UO}_{2}$, namely, $\mathrm{Y}^{3+}(1.02$ $\AA$ ionic radius in eightfold coordination) and $\mathrm{La}^{3+}(1.16 \AA)$ [24].

The paper is organized as follows. In the next section the details of the calculations are described. This is followed by a presentation of the results for formation energetics and defect interactions in Section 3. In Section 4 the results are discussed in the context of the previous models proposed for explaining the effect of cation size on the stability and defect ordering behavior in aliovalently doped fluorite structures.

\section{Computational Methodology}

The computational approach employed in this work is similar to that applied in studies of doped $\mathrm{ZrO}_{2}$ and $\mathrm{ThO}_{2}$ by Bogicevic et al. [8, 9] and Alexandrov et al. [11], respectively. The approach involves calculations of a relatively large number of ordered defect-dopant configurations as a basis

for exploring trends in energetic stability and defect ordering. The following subsections provide detailed descriptions of the structural enumeration techniques (Sec. 2.1), the screening of the structures using ionic pair potential models (Sec. 2.2), and the DFT based calculations (Sec. 2.3) implemented in this work.

\subsection{Structure Enumeration}

In order to determine the most stable defect structures for fluorite-structured $\mathrm{UO}_{2}$ doped with trivalent cation $\left(\mathrm{M}^{3+}\right)$ oxides, we investigate many hypothetical cation-vacancy arrangements. The cation sublattice contains host and dopant species (i.e., $\mathrm{U}^{4+}$ and $\mathrm{M}^{3+}$, respectively), and the anion sublattice contains host and vacancy species (i.e., $\mathrm{O}^{2-}$ and $\mathrm{O}_{v a c}$ ); the substitution of two $\mathrm{U}^{4+}$ by two $\mathrm{M}^{3+}$ can be charge compensated by one $\mathrm{O}_{v a c}$ ), as illustrated in Fig. 1. We employ a structure enumeration technique developed for 


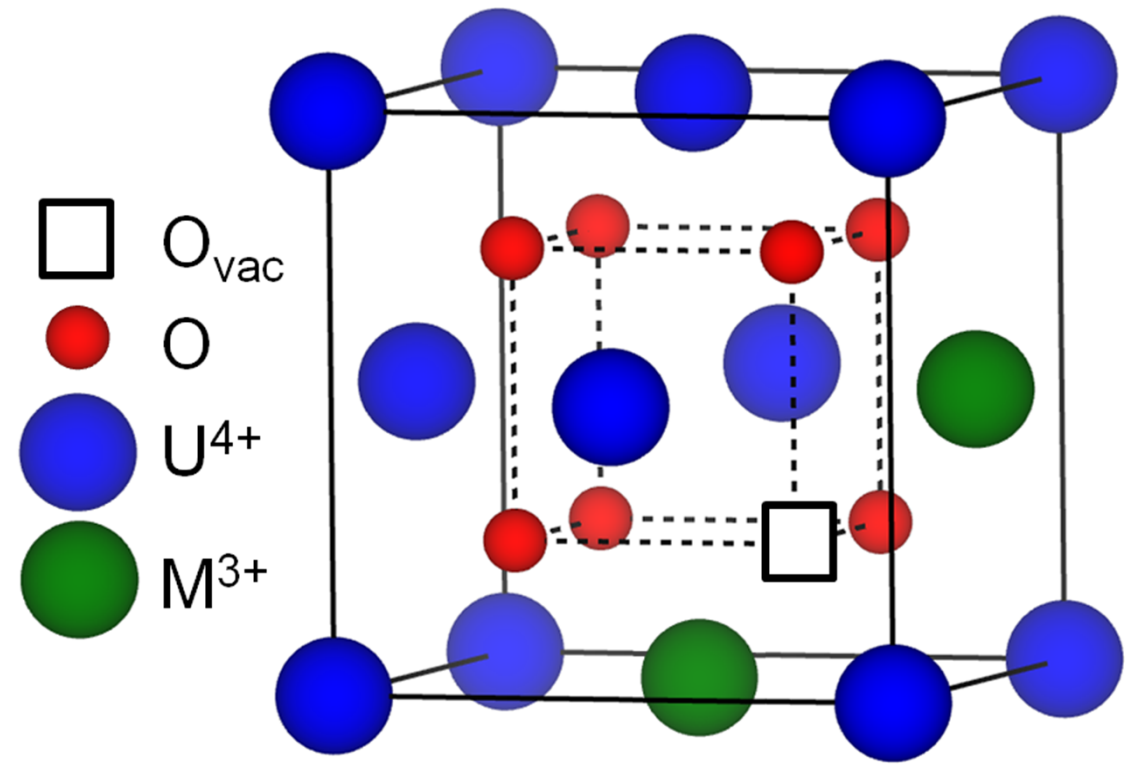

Figure 1: The ideal $\mathrm{UO}_{2}$ cubic fluorite structure with two trivalent dopants on the cation fcc sublattice and one charge compensating oxygen vacancy on an anion simple cubic sublattice is shown.

cluster expansion based studies of alloy thermodynamics, considering supercells consisting of up to six formula units, employing an algorithm by Hart and Forcade [25] that is implemented in the alloy theoretic automatic toolkit (ATAT) $[26,27]$. The compositions considered are $\mathrm{U}_{4} \mathrm{M}_{2} \mathrm{O}_{11}(\mathrm{x}=1 / 3$, six formula units: $4 \mathrm{U}$ ions, $2 \mathrm{M}$ ions, $11 \mathrm{O}$ ions, and $\left.1 \mathrm{O}_{v a c}\right), \mathrm{U}_{2} \mathrm{M}_{2} \mathrm{O}_{7}(\mathrm{x}=1 / 2$, four formula units: $2 \mathrm{U}$ ions, $2 \mathrm{M}$ ions, $7 \mathrm{O}$ ions, and $1 \mathrm{O}_{v a c}$ ), and $\mathrm{U}_{2} \mathrm{M}_{4} \mathrm{O}_{10}$ $\left(\mathrm{x}=2 / 3\right.$, six formula units: $2 \mathrm{U}$ ions, $4 \mathrm{M}$ ions, $10 \mathrm{O}$ ions, and $\left.2 \mathrm{O}_{\text {vac }}\right)$. The structure enumeration yielded 117,27 , and 710 symmetry-distinct structures for $\mathrm{x}=1 / 3,1 / 2$, and $2 / 3$, respectively.

\subsection{Classical Pair Potentials}

The energies of all enumerated structures were calculated using ionic pairpotential models as a first "screening" step, with the intent of eliminating from consideration by more computationally demanding DFT based calculations the electrostatically unfavorable structures for a given composition and $\mathrm{M}^{3+}$ species. In these calculations the potential energy between ions $i$ and $j$ 
is modeled through pair potentials of the following form:

$$
E\left(r_{i j}\right)=\frac{q_{i} q_{j}}{r_{i j}}+A \exp \left(\frac{-r_{i j}}{\rho}\right)-\frac{C}{r_{i j}^{6}}
$$

The first term represents the coulombic interaction where $q$ is the respective ionic charge and $r_{i j}$ is the distance between ions $i$ and $j$. The latter two repulsive and attractive terms represent the short range Buckingham potential, where $A, \rho$, and $C$ are system-dependent parameters. In addition, polarizabilities are introduced for individual ionic species according to the shell model as formulated by Dick and Overhauser [28]. The Buckingham and shell model parameters used in this work are given in Refs. [29, 30, 31]. Geometry relaxations and energy minimizations were performed using the General Utility Lattice Program [32].

\subsection{First-principles Calculations}

All structures for a given composition and $\mathrm{M}^{3+}$ species were ranked according to their total energy based on the ionic pair potential calculations described above. Initially DFT calculations were performed for the resulting six lowest energy structures for a given composition. If any of the five higher energy structures were found to be lower than the ground state predicted by the pair potential models, additional structures were considered in an effort to ensure that the lowest energy configuration was identified by the DFT based methods.

Most of the results reported below were obtained using DFT within the formalism of the projector augmented-wave (PAW) method [33, 34] and the Perdew-Burke-Ernzerhof (PBE) generalized gradient approximation (GGA) $[35,36]$ as implemented in the Vienna ab initio simulation package (VASP) $[37,38]$. In these calculations, a Hubbard-U correction was implemented within the formalism of Dudarev et al. [39] to account for the self-interaction error for localized $5 f$ electrons in uranium. The value of the parameter $\mathrm{U}_{\text {eff }}$ $=\mathrm{U}-\mathrm{J}$ in the DFT $+\mathrm{U}$ formalism was chosen to be $4.00 \mathrm{eV}$ for this study; this value is similar to that found to give optimal results in comparisons to spectroscopic data for $\mathrm{UO}_{2}$ by Dudarev et al. (3.99 eV) [39], and was found to provide good agreement between calculated and measured oxidation enthalpies of $\mathrm{UO}_{2}$ [40]. The PAW potentials used in this work are $\mathrm{U}, \mathrm{O}$, Y_sv, and La in the VASP PBE library. The PAW potentials use 14 valence electrons for $\mathrm{U}\left(6 \mathrm{~s}^{2} 6 \mathrm{p}^{6} 5 \mathrm{f}^{3} 6 \mathrm{~d}^{1} 7 \mathrm{~s}^{2}\right), 6$ for $\mathrm{O}\left(2 \mathrm{~s}^{2} 2 \mathrm{p}^{4}\right), 11$ for $\mathrm{Y}\left(4 \mathrm{~s}^{2} 4 \mathrm{p}^{6} 5 \mathrm{~s}^{2} 4 \mathrm{~d}^{1}\right)$, 
and 11 for La $\left(5 \mathrm{~s}^{2} 5 \mathrm{p}^{6} 6 \mathrm{~s}^{2} 5 \mathrm{~d}^{1}\right)$. We employ a plane-wave cutoff energy of 500 $\mathrm{eV}$, and the Brillouin zone is sampled using the Monkhorst-Pack scheme with at least $4 \times 4 \times 4$ k-point meshes. Atomic positions were relaxed with no symmetry constraints until residual forces were below approximately 20 $\mathrm{meV} / \AA$. From convergence checks with respect to plane-wave cutoff and $\mathrm{k}$ point sampling we estimate the formation enthalpies to be converged at the level of a few tenths of a $\mathrm{kJ} / \mathrm{mol}$-cation. The results of the first-principles calculations presented below made use of a scalar-relativistic approximation for valence electrons. We also performed calculations of the lowest energy $\mathrm{Y}$ and La-substituted structures for $\mathrm{x}=1 / 2$ including spin-orbit coupling, which was found to change the formation enthalpies by less than $1 \mathrm{~kJ} / \mathrm{mol}$-cation.

The formation enthalpy for a solid solution with respect to its constituent oxides is defined as

$$
\Delta H_{f}=E\left[U_{1-x} M_{x} O_{2-0.5 x}\right]-(1-x) E\left[U O_{2}\right]-x E\left[M O_{1.5}\right],
$$

where $\mathrm{E}\left[\mathrm{U}_{1-x} \mathrm{M}_{x} \mathrm{O}_{2-0.5 x}\right]$ denotes the energy per cation of a solid solution with cation dopant concentration $x, \mathrm{E}\left[\mathrm{UO}_{2}\right]$ denotes the energy per cation of fluorite-structured $\mathrm{UO}_{2}$ (calculated here using a 12-atom unit cell with $1 \mathrm{k}$ antiferromagnetic order), and $\mathrm{E}\left[\mathrm{MO}_{1.5}\right]$ denotes the energy per cation of $\mathrm{Y}_{2} \mathrm{O}_{3}$ or $\mathrm{La}_{2} \mathrm{O}_{3}$ in the experimentally observed (i.e., room temperature) cubic bixbyite (C-type sesquioxide) or hexagonal (A-type) structure, respectively. Both ferromagnetic and antiferromagnetic arrangements for the uranium ion magnetic moments were considered within the solid solution defect structures, and the latter were found to be lower in energy. Because there are four uranium atoms in the structures associated with $\mathrm{x}=1 / 3$, there are three unique antiferromagnetic spin arrangements (i.e., up-up-down-down, up-down-up-down, up-down-down-up). We found little difference in the calculated formation enthalpies with respect to each of these magnetic configurations (i.e., within $0.2 \mathrm{~kJ} /$ mol-cation).

In calculations that make use of the DFT $+\mathrm{U}$ methodology, it is well documented in the literature that one can converge to multiple metastable states corresponding to different orbital occupancies for the localized $f$ electrons $[41,42,43]$. Thus, a practical issue in such calculations is to ensure that the computed energy corresponds to the true electronic ground state (or close to it) $[44,45]$. Several different methods have been proposed to address this issue for $\mathrm{UO}_{2}[46,43,47,48,49]$. In this work we employ the approach by Meredig et al. [49], involving a gradual "ramping" up of the Hubbard-U parameter. In the current implementation, $\mathrm{U}_{\text {eff }}$ starts at zero and is increased 
stepwise by $0.1 \mathrm{eV}$ in order to localize the U $5 f$ electrons. This methodology offers sufficient computational efficiency for treating many compounds with relatively large unit cells. In order to be confident of the accuracy of the "Uramping" method, we compared results obtained by this approach with those derived by the occupation matrix control (OMC) method, which has been applied to plutonium oxides $\left(\mathrm{PuO}_{2}\right.$ and $\left.\mathrm{Pu}_{2} \mathrm{O}_{3}\right)$ [50] and subsequently $\mathrm{UO}_{2}$ $[47,48]$. The OMC approach involves initializing calculations with many distinct imposed values of the diagonal and off-diagonal occupation matrices for the $\mathrm{U} 5 f$ electrons, and taking the lowest of the resulting calculated energies as the ground-state.

For comparison of the "U-ramping" and OMC methods we performed static $\mathrm{DFT}+\mathrm{U}$ calculations of a simple defect-fluorite structure, namely, $\mathrm{ULa}_{2} \mathrm{O}_{5}$, which contains a single oxygen vacancy. We used the OMC approach discussed in Ref. [47] in which a total of 61 occupation matrices were sampled (21 diagonal, 40 off-diagonal). The ramping method gave the lowest total energy by approximately $2.4 \mathrm{~kJ} / \mathrm{mol}$-cation compared the lowest total energy determined using OMC. A more exhaustive sampling of matrices in the OMC approach could potentially result in energies that are closer to (or perhaps even lower than) that resulting from the "U-ramping" approach; however, it is encouraging that none of the energies derived from the OMC method were significantly lower than the value obtained from the ramping approach.

Separate DFT calculations using a hybrid functional [51] were implemented to compare results to formation enthalpies calculated using the $\mathrm{DFT}+\mathrm{U}$ method. The hybrid functional methodology has been shown to accurately describe lattice constants and electronic structure of $\mathrm{UO}_{2}[52,53,54,55,56]$. The "local hybrid functional for correlated electrons" (LHFCE), which is also referred to as "exact exchange for correlated electrons" (EECE), is a hybrid functional approach in which the exact exchange is applied locally within the atomic spheres only to the correlated electrons (i.e., $5 f$ uranium orbitals in the current work). The LHFCE functional is less computationally demanding compared to the full hybrid functional, and has been shown to accurately describe lattice constants, electronic structure, and defect formation energies of $\mathrm{UO}_{2}[57,58]$.

The LHFCE calculations were performed using the all-electron full- potential linearized augmented plane wave plus local orbitals basis (FP-L/APW+lo) method as implemented in the WIEN2k code. Details of the LHFCE method implementation in WIEN2k are discussed in Ref. [59]. The hybrid exchange- 
correlation energy functional used in this work has the form

$$
\Delta E_{X C}^{P B E+\alpha H F}=\alpha E_{X}^{H F}+(1-\alpha) E_{X}^{P B E}+E_{C}^{P B E},
$$

where $\alpha E_{X}^{H F}$ is the HF exchange, $E_{X}^{P B E}$ and $E_{C}^{P B E}$ are the PBE exchange and correlation energies, respectively, and $\alpha$ represents the fraction of $\mathrm{HF}$ exchange that replaces the PBE exchange. The value for $\alpha$ used in this work is 0.25 , which is representative of the PBE0 functional [60].

In the FP-L/APW+lo method the convergence of the basis set is controlled by a cutoff parameter $R_{r m t} K_{\max }$, where $R_{r m t}$ is the smallest atomic sphere radius in the cell and $K_{\max }$ is the magnitude of the largest wavenumber of the plane wave expansion. Additional local orbitals were added for $\mathrm{U} 6 \mathrm{~s}, 6 \mathrm{p}, 4 \mathrm{~d}, 5 \mathrm{f}$ and for $\mathrm{O} 2 \mathrm{~s}$ states. The $R_{r m t}$ values were set to $2.3 \mathrm{Bohr}$, 1.6 Bohr, 2.26 Bohr, and 2.3 Bohr for U, O, Y, and La, respectively, and $R_{r m t} K_{\max }=8$. The Brillouin zone was sampled with $32 \mathrm{k}$-points and the integrations were conducted by the tetrahedron method of Blöchl, Jepsen, and Andersen [33]. The calculations were performed spin polarized with antiferromagnetic configurations for the structures containing uranium atoms. The energy, charge, and force convergence criteria used for these computations are $1 \mathrm{meV}, 0.0001$ and $26 \mathrm{meV} / \AA$.

\section{Results}

The results for all calculations presented below were computed using the GGA + U method, unless explicitly stated otherwise. We first investigate the energetic stability of $\mathrm{U}_{1-x} \mathrm{M}_{x} \mathrm{O}_{2-0.5 x}(\mathrm{M}=\mathrm{Y}$, La) through consideration of the formation enthalpy $\Delta H_{f}$ with respect to constituent oxides, as defined in Eq. 2. Results for the lowest energy structures of $\mathrm{Y}$ and La-substituted $\mathrm{UO}_{2}$ are shown in Fig. 2 and Table 1 . The positive values of $\Delta H_{f}$ for Y-containing solid solutions indicate that phase separation is favored at low temperatures, whereas the negative values of $\Delta H_{f}$ for La-containing solid solutions indicate a compound forming tendency.

The formation enthalpy values for calculations with the hybrid functional are consistent with the DFT $+\mathrm{U}$ calculations for both $\mathrm{Y}$ and La-substituted systems, with respect to the order of magnitude and sign of $\Delta H_{f}$, and the trend with substitution level, as shown in Fig. 2. A comparison of the filled and open symbols, corresponding respectively to GGA+U and hybridfunctional results, show agreement at the level of a few $\mathrm{kJ} /$ mol-cation. This 
Table 1: Formation enthalpies (kJ/mol-cation) of $\mathrm{U}_{1-x} \mathrm{M}_{x} \mathrm{O}_{2-0.5 x}(\mathrm{M}=\mathrm{Y}, \mathrm{La})$ structures are listed for low-energy fully relaxed structures enumerated in this study. The structures listed have formation energies within $3 \mathrm{~kJ} / \mathrm{mol}$-cation of the lowest energy structure for each trivalent cation species at each composition considered. The fourth column lists the direction of ordering of oxygen vacancies. The last column lists the number of trivalent cations in the four tetrahedral nearest neighbor positions surrounding an oxygen vacancy. \# M-Vac

\begin{tabular}{ccrcc}
$\mathrm{x}$ & $\mathrm{M}$ & $\Delta H_{f}$ & Direction & Neighbors \\
\hline $2 / 3$ & La & -1.6 & $\langle 110\rangle$ & 2 \\
& $\mathrm{Y}$ & 10.8 & $\langle 110\rangle$ & 3 \\
& & 11.3 & $\langle 211\rangle$ & 3 \\
& & 12.3 & $\langle 211\rangle$ & 3 \\
& & 12.6 & $\langle 211\rangle$ & 3 \\
& & 12.6 & $\langle 110\rangle$ & 3 \\
$1 / 2$ & La & -1.3 & $\langle 110\rangle$ & 1 \\
& & 1.4 & $\langle 110\rangle$ & 1 \\
& & 2.1 & $\langle 110\rangle$ & 1 \\
& Y & 11.5 & $\langle 110\rangle$ & 3 \\
$1 / 3$ & La & -5.2 & $\langle 211\rangle$ & 0 \\
& Y & 7.5 & $\langle 211\rangle$ & 2 \\
& & 8.3 & $\langle 211\rangle$ & 2 \\
& & 8.9 & $\langle 211\rangle$ & 2 \\
& & 9.2 & $\langle 110\rangle$ & 3
\end{tabular}


good level of agreement between calculated results obtained using different codes and functionals is encouraging.

We consider next the relative ordering of the cation/vacancy configurations giving the lowest formation enthalpies. Table 1 lists, for the most stable configurations, the formation enthalpy and structural features including the direction of oxygen vacancy ordering and the number of trivalent cations (per vacancy site) that are nearest neighbors to an oxygen vacancy (for structures with $\mathrm{x}=2 / 3$ that contain two oxygen vacancies, the reported number of nearest neighbor cations is an average over both vacancy sites). In the fluorite lattice, each oxygen vacancy has four cation nearest neighbors, so that the maximum number of $\mathrm{M}^{3+}$ nearest neighbors to the vacancy site is four. We observe that the oxygen vacancies prefer to align as either second or sixth nearest neighbors along the $\langle 110\rangle$ and $\langle 112\rangle$ directions, respectively. In the lowest-energy structures for Y-substituted systems, a higher number of $\mathrm{M}^{3+}$ neighbors around the vacancies are preferred compared to the La-substituted systems for all compositions.

The formation enthalpies for all structures computed by the GGA+U method in this study are shown in Fig. 3. The wide range of enthalpies for each composition illustrates the magnitude of the dependence of the energy on the defect configuration. Y-substituted systems for $\mathrm{x}=1 / 3$ and $2 / 3$ show several nearly degenerate low energy structures, whereas the lowest-energy configuration for La-substituted systems shows a larger gap between the lowest and next-lowest energy structures for all three compositions. The results in Fig. 3 again highlight the stronger energetic preference for $\mathrm{M}^{3+}$ binding with oxygen vacancies in the Y-versus La-substituted systems.

\section{Discussion}

To gain additional insights into the factors governing the stability of trivalent impurities in $\mathrm{UO}_{2}$, we compare below the main results of the present work with those derived in previous computational and experimental studies of the energetics of aliovalently doped fluorite-structured oxide solid solutions $[11,8,3,4]$. Additionally, the nature and energetics of cation-vacancy ordering derived from the present results are analyzed, and the consequences of these findings for oxygen ion mobilities are discussed.

The formation enthalpies presented above for $\mathrm{UO}_{2}$-based solid solutions are lower than those computed in a previous DFT study of $\mathrm{ThO}_{2}$-based systems [11]. Considering that the ionic radius of $\mathrm{U}^{4+}(1.00 \AA)$ is smaller 


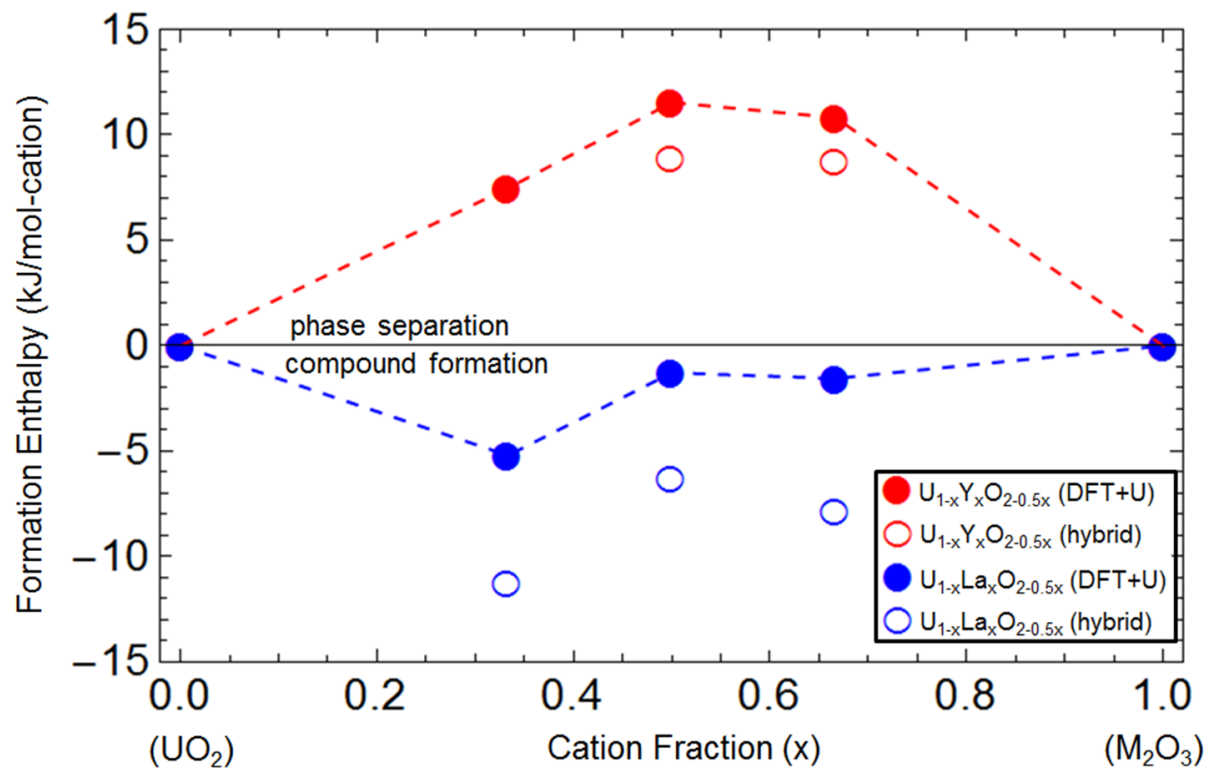

Figure 2: Formation enthalpies of $\mathrm{U}_{1-x} \mathrm{M}_{x} \mathrm{O}_{2-0.5 x}(\mathrm{M}=\mathrm{Y}$,La) structures are shown for the lowest-energy fully relaxed structures of all compositions enumerated in this study.

than that of $\mathrm{Th}^{4+}(1.05 \AA)$, these comparisons suggest a trend towards higher energetic stability (lower formation enthalpies) with decreasing size of the $4+$ host cation, for a given $\mathrm{M}^{3+}$ impurity. This trend is also observed if the present calculated values of $\Delta H_{f}$ for $\mathrm{Y}$-doped $\mathrm{UO}_{2}$ are compared with those derived in previous DFT calculations for Y-doped $\mathrm{ZrO}_{2}$ [8]; the latter are found to be significantly lower in energy, consistent with the smaller ionic radius of $\mathrm{Zr}^{4+}(0.84 \AA)$.

The effect of the $4+$ cation size is summarized for Y-doped solid solutions in Fig. 4 (similar behavior is also found for La-doping, although fewer results are available in the literature). This trend is consistent with observations derived from calorimetric experiments by Chen and Navrotsky [3] and Aizenshtein, Shvareva and Navrotsky [4]. In an analysis of the measured formation enthalpies across trivalent doped $\mathrm{ThO}_{2}, \mathrm{CeO}_{2}, \mathrm{ZrO}_{2}$ and $\mathrm{HfO}_{2}$, these authors have argued that the enhanced stability of systems with smaller $4+$ cations is associated with a preference for reduced oxygen ion coordination. Specifically, the formation of a fluorite-structured solid solution from $\mathrm{AO}_{2}$ and $\mathrm{M}_{2} \mathrm{O}_{3}$ constituents necessarily leads to a decrease in the average oxygen coordination surrounding the $\mathrm{A}^{4+}$ cation, relative to the eightfold coordina- 


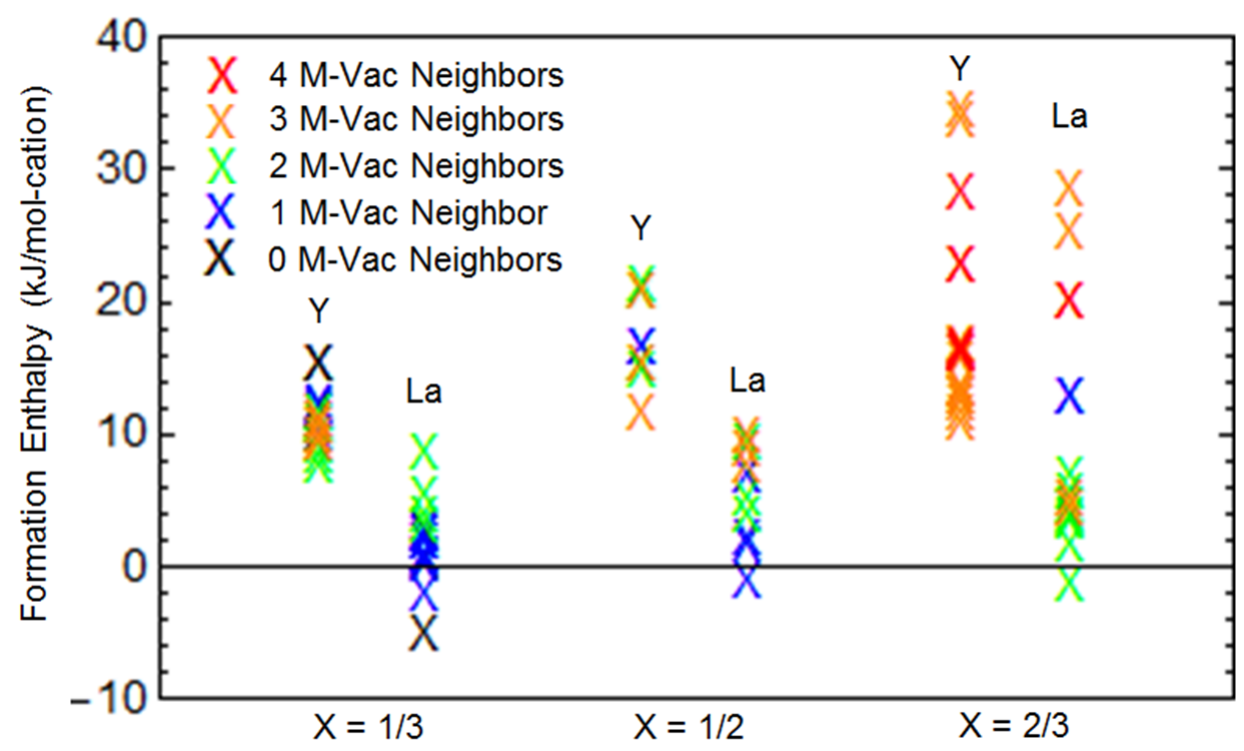

Figure 3: The formation enthalpies calculated using DFT $+\mathrm{U}$ for all structures computed in this study are shown. Each structure is denoted by the number of trivalent cations in the four tetrahedral nearest neighbor positions surrounding an oxygen vacancy according to the legend in the top left of the figure. Note that there are two vacancy sites for $x=2 / 3$, and the average number of neighbors between the two sites (rounded to the nearest neighbor) is shown. 
tion characteristic of the pure $\mathrm{AO}_{2}$ constituent. For small cations such as $\mathrm{Zr}^{4+}$ and $\mathrm{Hf}^{4+}$, which are known to prefer sevenfold coordination, this effect is expected to be stabilizing.

We consider next the trends in $\Delta H_{f}$ with respect to the size of the trivalent cation, for a fixed $4+$ cation species. Specifically, the trend towards more positive formation energies for the smaller $\mathrm{Y}$ versus the larger La trivalent cations is consistent with both calorimetric and computational results in $\mathrm{CeO}_{2}$ and $\mathrm{ThO}_{2}$ systems $[3,4,11]$. It has been suggested $[3,4]$ that this trend can be attributed to the smaller lattice distortions that have been observed to occur when larger trivalent cations are substituted into oxygen-vacancy charge-compensated fluorite-structured oxide compounds. Specifically, pair potential calculations of La- and Y-substituted $\mathrm{CeO}_{2}$ show much smaller changes in the nearest neighbor cation-cation nearest neighbor distances for La- relative to Y-substituted structures [61]. We find similar trends in the present work: La- $\mathrm{U}$ and $\mathrm{U}-\mathrm{U}$ distances in $\mathrm{U}_{0.5} \mathrm{La}_{0.5} \mathrm{O}_{1.75}$ structures are computed to be at most $0.08 \AA$ different from the nearest neighbor spacing on the cation sublattice of pure $\mathrm{UO}_{2}$ (the differences in bond distances are due to both volume change and local bond distortions), while for $\mathrm{U}_{0.5} \mathrm{Y}_{0.5} \mathrm{O}_{1.75}$ structures these bond-length distortions (i.e., Y-U, U-U) are as large as 0.20 A.

The DFT calculations are performed at $0 \mathrm{~K}$, whereas the calorimetric measurements, for which comparisons in systematic trends are made, correspond to room temperature. At finite temperature, additional contributions to the formation enthalpy arise (e.g., due to vibrational excitations) which have not been considered in the present work. To quantify the relative importance of these contributions, we consider a previous study [62] on Y-doped $\mathrm{UO}_{2}$. In this study it was reported that the heat capacities for the solidsolution samples showed approximately 30\% deviation from Kopp's law, i.e., from a concentration-weighted linear combination of the heat capacities of the constituent oxide components. We fit the heat capacity versus temperature data reported in [62] from $0-298 \mathrm{~K}$ to a third order polynomial, and integrated over this temperature range to compute a conservative estimate of the thermal contributions to the enthalpy at room temperature. From this value we subtracted the contribution to the enthalpies of the constituent compounds, given by integrating the Kopp's law heat capacity. The resulting estimate of the thermal contribution to the formation enthalpy at $298 \mathrm{~K}$ is approximately $0.3 \mathrm{~kJ} / \mathrm{mol}$-cation. This contribution is roughly an order of magnitude smaller than the formation enthalpies reported in this work. 
Therefore, we conclude that thermal effects lead to minimal contributions to the measured formation enthalpies at room temperature, and that their neglect in the DFT calculations is warranted for the purposes of the present study.

An additional consideration that should be addressed in the comparison of DFT calculations to measured formation enthalpies relates to the nature of the configurational order in experiments versus calculations. For the systems considered in this study, the experimental samples are known to be solid solutions, and lack long-range order characteristic of the compounds considered in the DFT calculations. Comparisons between such DFT calculations and experiments can be justified if the latter display appreciable short-range order. While substantial ordering effects have been reported in experimental studies on similar fluorite-structured solid solutions $[4,1,2,3]$, it is difficult to quantify whether the effects are sufficiently pronounced for direct comparison with DFT calculations on fully ordered compounds. Nevertheless, it is assumed that the trends with respect to trivalent cation species should be comparable regardless of the extent of order/disorder, as was found to be the case in previous studies for La and Y substituted $\mathrm{ThO}_{2}$. Specifically, it was found that the energetic trends for $\mathrm{ThO}_{2}$ with respect to dopant size are consistent whether based on DFT results for ordered systems [11], experimental measurements for samples with some degree of configurational short-range order, or estimates of the energetics of fully disordered solid solutions derived from experimental data [4]. Since such trends are the focus of the current work, we believe comparisons between experimental measurements and DFT calculations are justified.

We consider finally an analysis of the nature of the energetically favorable cation-vacancy ordering tendencies for the $\mathrm{UO}_{2}-\mathrm{M}_{2} \mathrm{O}_{3}$ structures, considering first the lowest-energy structure of composition $\mathrm{U}_{2} \mathrm{La}_{2} \mathrm{O}_{7}(\mathrm{x}=1 / 2)$, which is computed to have a negative formation enthalpy in the present study, suggesting a tendency towards stable compound formation. We focus on this particular stoichiometry, as it is characteristic of the pyrochlore structure $\left(\mathrm{A}_{2} \mathrm{~B}_{2} \mathrm{O}_{7}\right)$ which is found to be a thermodynamically stable compound for many fluorite-based systems [63]. The pyrochlore structure is a derivative of fluorite where the A and B cations are ordered along the $\langle 110\rangle$ direction, and the tetrahedrally-coordinated oxygen vacancies are surrounded by adjacent $\mathrm{A}^{4+}$ cations. The configuration of the pyrochlore structure requires eight formula units ( $4 \mathrm{U}$ ions, 4 La ions, $14 \mathrm{O}$ ions, and $2 \mathrm{O}$ vac), and was therefore not considered in our structure enumerations, which considered 


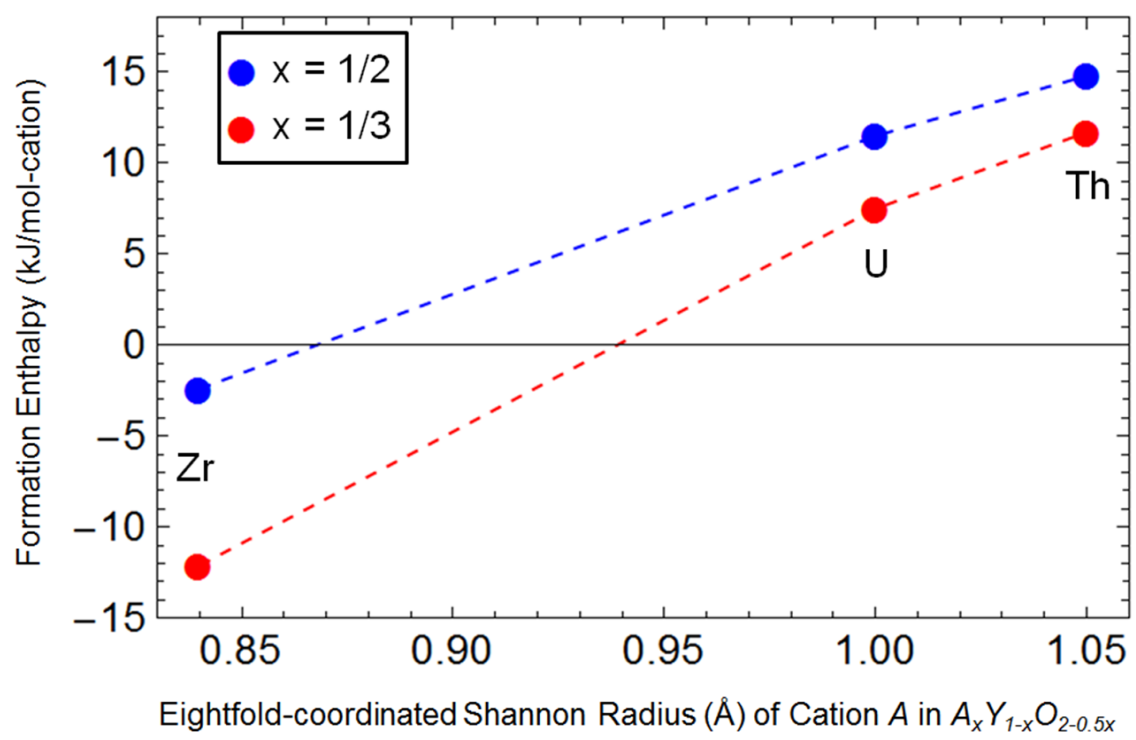

Figure 4: Calculated formation enthalpies for Y-substituted fluorite systems are shown. Results for zirconia $(\mathrm{A}=\mathrm{Zr})$ and thoria $(\mathrm{A}=\mathrm{Th})$ systems were taken from Refs. [8] and [11], respectively.

compounds with four formula units for $\mathrm{x}=1 / 2$. However, we performed an additional calculation of the formation enthalpy for pyrochlore-structured $\mathrm{U}_{2} \mathrm{La}_{2} \mathrm{O}_{7}$, obtaining a value of $\Delta H_{f}=+5.8 \mathrm{~kJ} /$ mol-cation, which is roughly $7 \mathrm{~kJ} /$ mol-cation higher in energy than the lowest-energy configuration for $\Delta H_{f}$ identified in this work. The relatively high energy of the pyrochlore structure for this system is consistent with arguments suggested previously based on the $\mathrm{B}^{3+} / \mathrm{A}^{4+}$ cation size ratios required for pyrochlore stability [63].

To gain insight into the nature of the preferred interactions between the trivalent dopants and charge-compensating oxygen vacancies in $\mathrm{UO}_{2}-\mathrm{M}_{2} \mathrm{O}_{3}$ solid solutions, we plot in Fig. 5 formation enthalpies for three structures featuring different number of trivalent cation/oxygen vacancy nearest neighbors, for both $\mathrm{M}=\mathrm{La}$ and $\mathrm{Y}$. The three structures considered have the same vacancy ordering pattern (i.e., $\langle 110\rangle$ ) and one, two or three $\mathrm{M}^{3+}$-vacancy neighbors. The configurations chosen are 1) the lowest energy Y-substituted structure (three $\mathrm{M}^{3+}-\mathrm{O}_{\text {vac }}$ neighbors), 2) the lowest energy La substituted structure (one $\mathrm{M}^{3+}-\mathrm{O}_{\text {vac }}$ neighbor), and 3) a structure with two $\mathrm{M}^{3+}$-vacancy neighbors that is within $4 \mathrm{~kJ} / \mathrm{mol}$-cation of the lowest energy structure for both La- and Y-substituted systems. 
The results in Fig. 5 demonstrate that $\mathrm{Y}$ and La dopants display qualitatively different preferences; the formation enthalpies for Y- and La- substituted structures decrease (more exothermic) and increase (more endothermic), respectively, with respect to the number of $\mathrm{M}^{3+}$-vacancy neighbors. These results suggest a significant energetic preference for $\mathrm{M}^{3+}$-oxygen vacancy and $\mathrm{U}^{4+}$-oxygen vacancy clustering in $\mathrm{Y}$ and La containing systems, respectively. In both cases, these clustering tendencies would be expected to lower oxygen-ion mobility, as discussed previously in the context of ionic conductivity in fluorite-structured solid electrolytes [13]. The current results thus support the suggestion from Ref. [14] that the effect of increased burnup (leading to higher concentrations of soluble trivalent fission products) on oxidative corrosion rates in spent nuclear fuel can be attributed to lower oxygen mobility due to defect association.

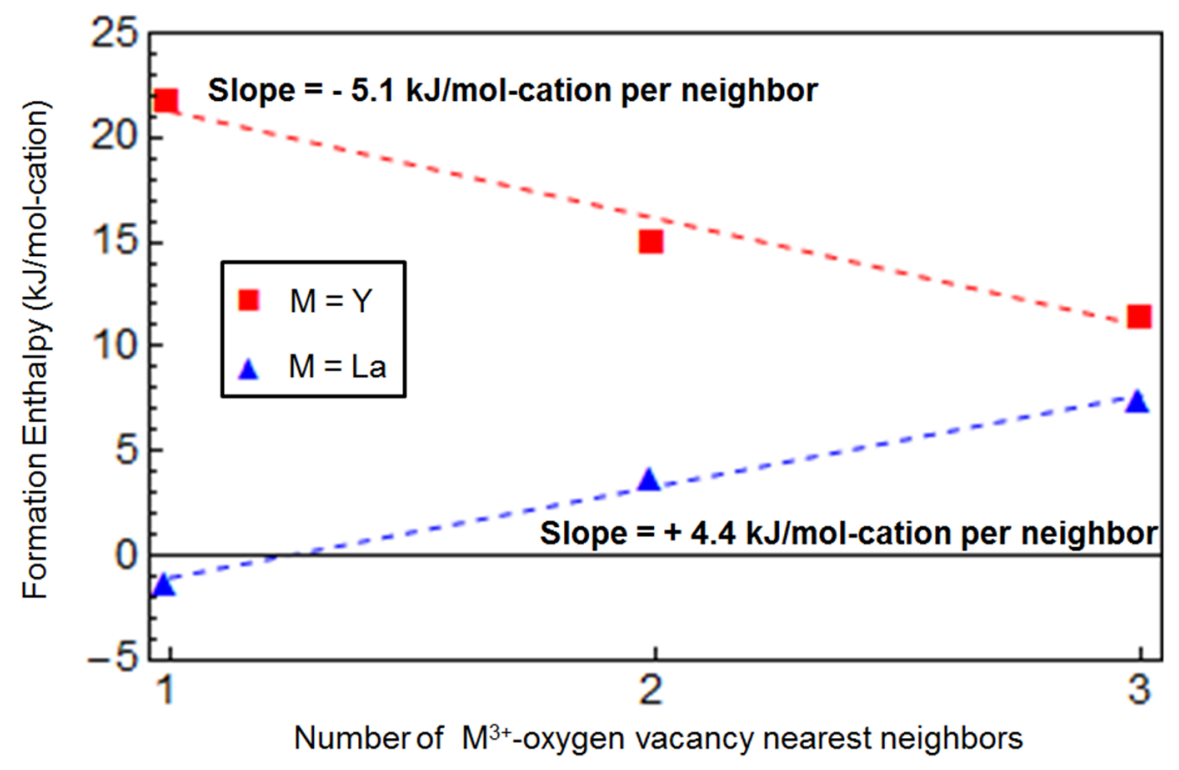

Figure 5: The formation enthalpies for selected structures containing, one, two, and three trivalent cation nearest neighbors to an oxygen vacancy are shown for $\mathrm{Y}$ and La-substituted systems at $\mathrm{x}=1 / 2$.

\section{Conclusions}

In this study DFT based computational methods were employed to study formation energetics and defect ordering tendencies in $\mathrm{UO}_{2}$ compounds sub- 
stituted with Y and La cations, which are common soluble fission products in nuclear fuel. We consider substitutional configurations that are chargecompensated with oxygen vacancies, finding that phase separation is energetically favored for all compositions considered for $\mathrm{Y}$-substituted $\mathrm{UO}_{2}$, whereas compound formation is favored for La-substituted $\mathrm{UO}_{2}$. The calculations are thus characterized by a trend towards increasing energetic stability of solid solutions with the larger La impurity relative to the smaller Y impurity. This trend is consistent with previous computational and calorimetric studies $[3,4,11]$ for fluorite-structured solid solutions based on $\mathrm{CeO}_{2}$ and $\mathrm{ThO}_{2}$.

We observe in general that oxygen vacancies prefer to align as either second or sixth nearest neighbors on the oxygen sublattice along the $\langle 110\rangle$ and $\langle 112\rangle$ directions, respectively, suggesting that the energetically preferred defect ordering configurations are characterized by a repulsive interaction between nearest neighbor oxygen vacancies. In the Y-substituted systems, structures with an enhanced number of vacancies around the $\mathrm{Y}^{3+}$ ions are found to be favored energetically, while the opposite is found for La-substituted systems. The pronounced energetic preferences for dopant cation-vacancy or host cation-vacancy clustering are expected to have an important effect on oxygen ion mobilities and therefore the oxidative corrosion rates in spent nuclear fuel.

\section{Acknowledgments}

J.M. Solomon was supported by the Office of Basic Energy Sciences of the U.S. Department of Energy as part of the Materials Science of Actinides Energy Frontier Research Center (DE-SC0001089) for initial DFT+U calculations, the U.S. Department of Energy through the Lawrence Livermore National Laboratory (DE-AC52-07NA27344) for the hybrid calculations, and the Department of Defense through the National Defense Science \& Engineering Graduate Fellowship Program for the remainder of the work. V. Alexandrov, A. Navrotsky, and M. Asta were supported by the U.S. Department of Energy as part of the Materials Science of Actinides Energy Frontier Research Center (DE-SC0001089). B. Sadigh was supported by the U.S. Department of Energy through the Lawrence Livermore National Laboratory (DE-AC52-07NA27344). This work made use of resources of the National

Energy Research Scientific Computing Center, supported by the Office of Basic Energy Sciences of the U.S. Dept of Energy (DE-AC02-05CH11231). 
The authors would like to thank B. E. Hanken and L. Zhang for useful discussions.

[1] T. A. Lee, A. Navrotsky, I. Molodetsky, Journal of Materials Research 18 (2003) 908-918.

[2] T. A. Lee, A. Navrotsky, Journal of Materials Research 19 (2004) 18551861.

[3] W. Chen, A. Navrotsky, Journal of Materials Research 21 (2006) 32423251.

[4] M. Aizenshtein, T. Y. Shvareva, A. Navrotsky, Journal of the American Ceramic Society 93 (2010) 4142-4147.

[5] A. K. A. Pryde, S. Vyas, R. W. Grimes, J. A. Gardner, R. Wang, Phys. Rev. B 52 (1995) 13214-13222.

[6] M. S. Khan, M. S. Islam, D. R. Bates, J. Mater. Chem. 8 (1998) 22992307.

[7] L. Minervini, M. O. Zacate, R. W. Grimes, Solid State Ionics 116 (1999) $339-349$.

[8] A. Bogicevic, C. Wolverton, G. M. Crosbie, E. B. Stechel, Phys. Rev. B 64 (2001) 014106.

[9] A. Bogicevic, C. Wolverton, Phys. Rev. B 67 (2003) 024106.

[10] A. Predith, G. Ceder, C. Wolverton, K. Persson, T. Mueller, Phys. Rev. B 77 (2008) 144104.

[11] V. Alexandrov, N. Grønbech-Jensen, A. Navrotsky, M. Asta, Phys. Rev. B 82 (2010) 174115.

[12] M. O. Zacate, L. Minervini, D. J. Bradfield, R. W. Grimes, K. E. Sickafus, Solid State Ionics 128 (2000) $243-254$.

[13] H. Inaba, H. Tagawa, Solid State Ionics 83 (1996) 1 - 16.

[14] H. He, P. G. Keech, M. E. Broczkowski, J. J. Nol, D. W. Shoesmith, Canadian Journal of Chemistry 85 (2007) 702-713. 
[15] L. Thomas, R. Einziger, H. Buchanan, Journal of Nuclear Materials 201 (1993) $310-319$.

[16] J.-W. Choi, R. J. McEachern, P. Taylor, D. D. Wood, Journal of Nuclear Materials 230 (1996) $250-258$.

[17] J. Cobos, D. Papaioannou, J. Spino, M. Coquerelle, Journal of Alloys and Compounds 271273 (1998) $610-615$.

[18] R. McEachern, P. Taylor, Journal of Nuclear Materials 254 (1998) 87 121.

[19] M. E. Torrero, E. Baraj, J. de Pablo, J. Gimnez, I. Casas, International Journal of Chemical Kinetics 29 (1997) 261-267.

[20] D. Shoesmith, Journal of Nuclear Materials 282 (2000) 1 - 31.

[21] D. Shin, T. M. Besmann, Journal of Nuclear Materials 433 (2013) 227 - 232. And references therein.

[22] L. Mazeina, A. Navrotsky, M. Greenblatt, Journal of Nuclear Materials 373 (2008) $39-43$.

[23] S. Middleburgh, D. Parfitt, R. Grimes, B. Dorado, M. Bertolus, P. Blair, L. Hallstadius, K. Backman, Journal of Nuclear Materials 420 (2012) 258 $-261$.

[24] R. D. Shannon, Acta Crystallographica Section A 32 (1976) 751-767.

[25] G. L. W. Hart, R. W. Forcade, Phys. Rev. B 77 (2008) 224115.

[26] A. van de Walle, M. Asta, G. Ceder, Calphad 26 (2002) 539 - 553.

[27] A. van de Walle, Calphad 33 (2009) 266 - 278.

[28] B. G. Dick, A. W. Overhauser, Phys. Rev. 112 (1958) 90-103.

[29] D. J. Binks, R. W. Grimes, Journal of the American Ceramic Society 76 (1993) 2370-2372.

[30] G. Busker, A. Chroneos, R. W. Grimes, I.-W. Chen, Journal of the American Ceramic Society 82 (1999) 1553-1559. 
[31] R. W. Grimes, G. Busker, M. A. McCoy, A. Chroneos, J. A. Kilner, S. P. Chen, Berichte der Bunsengesellschaft fur physikalische Chemie 101 (1997) 1204-1210.

[32] J. D. Gale, A. L. Rohl, Molecular Simulation 29 (2003) 291-341.

[33] P. E. Blöchl, Phys. Rev. B 50 (1994) 17953-17979.

[34] G. Kresse, D. Joubert, Phys. Rev. B 59 (1999) 1758-1775.

[35] J. P. Perdew, K. Burke, M. Ernzerhof, Phys. Rev. Lett. 77 (1996) 38653868.

[36] J. P. Perdew, K. Burke, M. Ernzerhof, Phys. Rev. Lett. 78 (1997) 13961396.

[37] G. Kresse, J. Furthmüller, Phys. Rev. B 54 (1996) 11169-11186.

[38] G. Kresse, J. Furthmller, Computational Materials Science 6 (1996) 15 -50 .

[39] S. L. Dudarev, G. A. Botton, S. Y. Savrasov, C. J. Humphreys, A. P. Sutton, Phys. Rev. B 57 (1998) 1505-1509.

[40] B. E. Hanken, C. R. Stanek, N. Grønbech-Jensen, M. Asta, Phys. Rev. B 84 (2011) 085131.

[41] B. Dorado, M. Freyss, B. Amadon, M. Bertolus, G. Jomard, P. Garcia, Journal of Physics: Condensed Matter 25 (2013) 333201. And references therein.

[42] F. Zhou, V. Ozolinšs, Phys. Rev. B 80 (2009) 125127.

[43] F. Zhou, V. Ozolinšs, Phys. Rev. B 83 (2011) 085106.

[44] A. Shick, W. Pickett, A. Liechtenstein, Journal of Electron Spectroscopy and Related Phenomena 114116 (2001) 753 - 758.

[45] P. Larson, W. R. L. Lambrecht, A. Chantis, M. van Schilfgaarde, Phys. Rev. B 75 (2007) 045114.

[46] H. Y. Geng, Y. Chen, Y. Kaneta, M. Kinoshita, Q. Wu, Phys. Rev. B $82(2010) 094106$. 
[47] B. Dorado, B. Amadon, M. Freyss, M. Bertolus, Phys. Rev. B 79 (2009) 235125.

[48] B. Dorado, G. Jomard, M. Freyss, M. Bertolus, Phys. Rev. B 82 (2010) 035114.

[49] B. Meredig, A. Thompson, H. A. Hansen, C. Wolverton, A. van de Walle, Phys. Rev. B 82 (2010) 195128.

[50] G. Jomard, B. Amadon, F. m. c. Bottin, M. Torrent, Phys. Rev. B 78 (2008) 075125.

[51] A. D. Becke, 98 (1993) 5648-5652.

[52] K. N. Kudin, G. E. Scuseria, R. L. Martin, Phys. Rev. Lett. 89 (2002) 266402.

[53] I. D. Prodan, G. E. Scuseria, R. L. Martin, Phys. Rev. B 73 (2006) 045104.

[54] L. E. Roy, T. Durakiewicz, R. L. Martin, J. E. Peralta, G. E. Scuseria, C. G. Olson, J. J. Joyce, E. Guziewicz, Journal of Computational Chemistry 29 (2008) 2288-2294.

[55] X.-D. Wen, R. L. Martin, L. E. Roy, G. E. Scuseria, S. P. Rudin, E. R. Batista, T. M. McCleskey, B. L. Scott, E. Bauer, J. J. Joyce, T. Durakiewicz, Journal of Chemical Physics 137 (2012) 154707.

[56] X.-D. Wen, R. L. Martin, G. E. Scuseria, S. P. Rudin, E. R. Batista, The Journal of Physical Chemistry C 117 (2013) 13122-13128.

[57] F. Jollet, G. Jomard, B. Amadon, J. P. Crocombette, D. Torumba, Phys. Rev. B 80 (2009) 235109.

[58] J.-P. Crocombette, D. Torumba, A. Chartier, Phys. Rev. B 83 (2011) 184107.

[59] P. Novak, J. Kune, L. Chaput, W. E. Pickett, Physica Status Solidi (b) 243 (2006) 563-572.

[60] C. Adamo, V. Barone, The Journal of Chemical Physics 110 (1999). 
[61] H. Hayashi, R. Sagawa, H. Inaba, K. Kawamura, Solid State Ionics 131 (2000) $281-290$.

[62] M. Beauvy, T. Duverneix, C. Berlanga, R. Mazoyer, C. Duriez, Journal of Alloys and Compounds 271273 (1998) 557 - 562.

[63] M. Subramanian, G. Aravamudan, G. S. Rao, Progress in Solid State Chemistry 15 (1983) $55-143$. 